Aus der I. chirurgischen Abteilung des R. Virchow-Krankenhauses (Direktor: Prof. Hermes).

\title{
Inkomplete Harnblasenruptur.
}

Von Dr. Moritz Cohn, Assistenzarzt.

(Mit 2 Abbildungen).

Obwohl in den letzten Jahren eine ganze Reihe von kompleten Harnblasenrupturen mit Glück und Erfolg operiert worden ist, muß man eine Blasenruptur, mag sie intraperitoneal oder extraperitoneal sitzen, noch immer als eine lebensgefährliche Verletzung bezeichnen. Inkomplete, subperitoneale Harnblasenrupturen hält man im allgemeinen für harmlos und legt ihnen keine große Bedeutung bei. Diese Anschauung findet sich auch im Handbuch der praktischen Chirurgie von Bergmann, Mikulicz und Bruns vertreten. Nur Seldowitsch ${ }^{1}$ ) (I904) und auf diesen zurückgreifend $\mathrm{N}$ or d $\mathrm{m} \mathrm{a} \mathrm{n}^{2}$ ) (Ig08) stellen die Forderung auf, daß man die unvollständige Blasenruptur wie eine vollständige zu behandeln habe. Uber das klinische Bild lassen sich beide nicht aus. Und doch dürfte es von Wert sein, differentialdiagnostisch das Bild der kompleten Ruptur der Harnblase von dem der unvollständigen, subperitonealen abzugrenzen.

In der Literatur, insbesondere der neueren, finden sich nur ganz spärliche Mitteilungen über derartige Verletzungen. Jedenfalls hat die richtige Diagnose bisher niemanden zum operativen sofortigen Eingreifen veranlaßt.

Im folgenden möchte ich über einen Fall von unvollständiger, subperitonealer Blasenruptur berichten, der geeignet ist, die An-

r) Seldowitsch, Uber intraperitoneale Rupturen der Harnblase. Archiv f. khn. Chirurgie, Bd. 72 .

2) Nordmann, Intraperitoneale Ruptur der Harnblase. Deutsche med. Wochenschrift 1908 . 
nahme, daß inkomplete Blasenrupturen harmlos seien, umzustoßen. Vier Stunden nach dem Trauma stand in diesem Falle für uns die Diagnose unvollständige Blasenruptur fest; wir nahmen aber, von obiger Ansicht geleitet, zunächst von einer sofortigen Laparotomie Abstand und beschlossen erst dann einzugreifen, wenn bedrohliche Erscheinungen eintreten sollten.

Der Arbeiter W., 42 Jahre alt, wurde eine halbe Stunde vor seiner Aufnahme in das Krankenhaus von einer elektrischen Bahn, während er auf seinem Rade saß, angefahren und geriet dabei unter die Vorderplattform des Wagens. Von der Feuerwehr wurde er aus dieser Lage befreit. Eine halbe Stunde vor dem Unfall hat er zuletzt Urin gelassen.

Befund: Kräftiger Mann, blaß, im Choc. Die Kleider sind entsprechend dem rechten Hüftbeinkamm und Schambeinrand mit Straßenstaub bedeckt. Puls 8o, Temp. 37. Leukozyten $x_{5}$ 000, Herz und Lungen ohne krankhafte Veränderungen. Abdomen weich, nirgends gespannt. Leber- und Nierengegend frei. Nur dicht über der rechten Leistenpforte findet sich ein kleinhandtellergroßer, druckempfindlicher $\mathrm{Be}$ zirk. Druck auf beide Darmbeinkämme löst keine Schmerzen aus. Per rectum: Rechts hoch oben druckempfindliche Stelle. - Dranggefühl zum Urinlassen. - Über beiden Brachioradialwülsten Hautabschürfungen. - Wirbelsäule unversehrt. Pat. kann laufen. Keinerlei Störungen von seiten des Nervensystems.

3 Stunden später: Patient ist unruhig; es ist ihm übel. Starker Drang zum Urinlassen, ohne daß es ihm möglich wäre, spontan Wasser zu lassen. Diffuse Ba u chdeckenspannung. Starke Schmerzhaftigkeit zweifingerbreit über der rechten Lejstenpforte in kleinhandtellergroßem Bezirk. Beim Katheterisieren entleeren sich etwa $60-70 \mathrm{ccm}$ fast reines Blut aus der Blase. $-\mathrm{D}$ a $\mathrm{n}$ a $\mathrm{ch}$ wir $\mathrm{r}$ $\mathrm{d}$ e r B a u $\mathrm{ch}$ wi e der w e i c h und bleibt es längere Zeit. - Weitere 3 Stunden später: Spannung der rechten Bauchseite. Leichter Meteorismus. - Weitere 5 Stunden später: Rechtsseitige Bauchdeckenspannung, Meteorismus. - Weitere 5 Stunden später: Diffuse Auftreibung des Leibes, rechts unten besonders schmerzhaft. Harndrang. Durch Katheterisieren werden $250 \mathrm{ccm}$ fast klarer Urin gewonnen. Danach wird der Leib weich, die Auftreibung ist geschwunden.

7 Stunden später: Durch Katheterisieren werden $50 \mathrm{ccm}$ klarer Urin entleert. $21 / 2$ Stunden später läßt Patient spontan $50 \mathrm{ccm}$ klaren Urin. 5 Stunden später entleert Patient wieder $30 \mathrm{ccm}$ Urin spontan. Rechtsseitige Bauchdeckenspannung. Beginnender Meteorismus. - 9 Stunden später: Patient hat in der $Z$ wischenzeit $30 \mathrm{ccm}$ Urin gelassen. Katheterisieren: qo $\mathrm{ccm}$ klarer Urin. Starker Meteorismus, Druckempfindlichkeit rechts neben der Blase nach der Ileocöcalgegend zu. Druckempfindlichkeit der rechten Nierengegend. - 5 Stunden später: Starker Meteorismus des ganzen Bauches. Die Druckempfindlichkeit reicht 
bis zum Nabel. Hormonal 15,0 intravenös. 20 Minuten später Schüttelfrost. - 3 Stunden später: Magenspülen. Dabei gehen Winde und etwas dünnflüssiger Kot $\mathrm{ab}$. -4 Stunden später: Operation (Prof. Hermes). ÄtherChloroform - Sauerstoff - Narkose. Schnitt in die Mittellinie vom Nabel bis zur Symphyse. Das prävesikale Gewebe ist stark ödematös durchtränkt. Eröffnen des Peritoneums. Därme injiziert, leicht gebläht. Sanguinolente Flüssigkeit im Bauch. - Steilste Beckenhochlagerung. - Abstopfen. Es gehen Winde $a b$. - Nun zeigt die rechte Hälfte der Blasenrückwand in fast Fünfmarkstückgröße eine graubläuliche Verfärbung. Diese Verfärbung

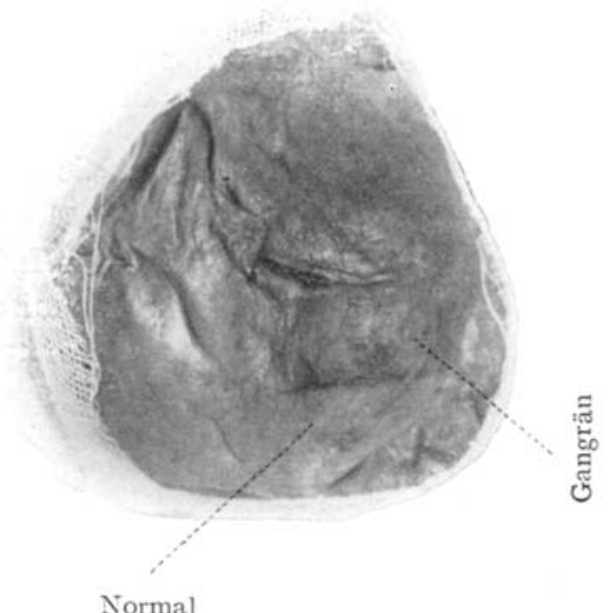

Normal scheint sich auch $I_{1,2}^{1,} \mathrm{~cm}$, weit auf den untersten Ureterabschnitt zu erstrecken. Diese Verfärbung muß als beginnende Gangrän angesprochen werden. Von der partiellen Blasenresektion wird wegen der bestehenden Peritonitis abgesehen. Beuteltampon in den Douglas. - Zwei durchgreifende Nähte über zwei Ballentupfern. Danerkatheter. Patient erholt sich nicht mehr, unter den Erscheinungen der diffusen Peritonitis verfällt er mehr und mehr. 36 Stunden nach der Operation tritt der Exitus ein.

Sektion (gerichtlich, Prof. Straßmann): Diffuse Peritonitis. In der rechten Hälfte der Blasenrückwand liegt eine fast fünfmarkstückgroße, graugrünlich verfärbte Stelle. Die Serosa ist hier scheinbar abgehoben und in Falten gelegt (vgl. Abbildung Fig. I). Der obere Rand dieser

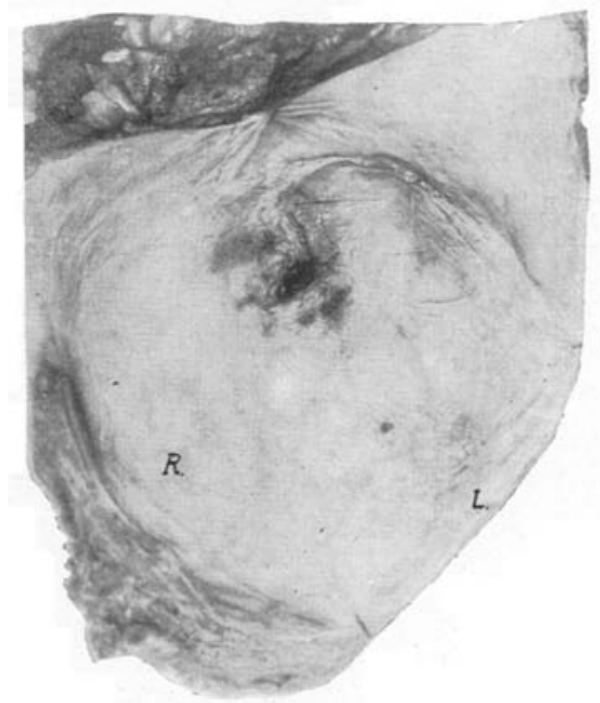

Fig. 2. Blaseninnenfläche. 
nekrotischen Partie liegt etwa I $1 / 2$ Querfinger breit unter dem Blasenscheitel. Dem medialen oberen Quadranten dieses Bezirkes entsprechend liegt an der Schleimhaut der Blase ein $12 \mathrm{~mm}$ langer RiB, der die Muscularis bis auf $3 \mathrm{~mm}$ Tiefe durchsetzt. Der Muscularisriß hat die Länge von $\mathrm{I} \mathrm{cm}$. Oberhalb und unterhalb des Risses finden sich mehrere punkt- und strichförmige Suffusionen (vgl. Abbildung Fig. 2). linien.

Am rechten horizontalen Schambeinast finden sich zwei Sprung-

Dieser Fall bietet manches Interessante. Zunächst in diagnostischer Hinsicht! - Das klinische Bild der intraperitonealen Blasenruptur wird von zwei Komponenten gebildet. Es sind dies einerseits Erscheinungen, welche auf Funktionsstörungen des verletzten Organes selbst hinweisen, andererseits sind es Abdominalerscheinungen allgemeinerer Natur, die darauf hindeuten, daß fremder Inhalt - Kot, Blut, Urin, Galle und ähnliches - in die freie Bauchhöhle getreten ist, und daß sich hier ein pathologischer Prozeß abspielt. Klagt ein Patient, der eine Verletzung des Unterbauches erlitten hat, über häufigen Harndrang, ohne daß es ihm spontan möglich wäre, Urin zu lassen, istErbrechen aufgetreten, besteht eine deutliche Bauchdeckenspannung, so muß man eine an Blasenruptur denken. Entleert man ferner beim Katheterisieren zunächst Blut, resp. etwas mit Blut stark vermischten Urin oder gelangt man beim Katheterisieren zufällig durch der: Riß in die Bauchhöhle und fühlt die Katheterspitze in Nabelhöhe unter der Bauchdecke, so dürfte die Diagnose sicher sein! So scharf und deutlich findet sich das klinische Bild meist nur in den Fällen, die kurze Zeit oder wenige Stunden nach der Verletzung in ärztliche Beobachtung kommen. Ist dagegen längere Zeit seit dem Unfall vergangen, so treten die Erscheinungen, welche für die Verletzung der Blase charakteristisch sind, in den Hintergrund und die Erscheinungen der Bauchfellentzündung beherrschen das Krankheitsbild.

Bei der subperitonealen Blasenruptur hat man theoretisch nur die Symptome zu erwarten, die durch die Verletzung der Blase selbst ausgelöst werden: der häufige Harndrang, die Unmöglichkeit, Urin lassen zu können und die Fntleerung von Blut, resp. stark blutigen Urins beim Katheterisieren.

Dringt kein Urin in die Bauchhöhle, wird das über der Riß- 
stelle liegende Peritoneum nicht durch die in der Blase sich ansammelnde Blut- und Urinmenge entfaltet und gedehnt oder dadurch gereizt, daß sich die Subserosa mit Blut oder Urin imbibiert, so wird man die Bauchdeckenspannung vermissen.

Diesen theoretischen Erwägungen entspricht ganz das klinische Bild in obigem Falle. Der Bauch ist weich und überall tief eindrückbar, nur rechts dicht über dem inneren Leistenring - am Orte der Verletzung - besteht eine gewisse Schmerzhaftigkeit, der Patient erbricht nicht, die charakteristischen Funktionsstörungen dagegen, die auf eine Blasenverletzung hinweisen, - Urindrang und die Unmöglichkeit Urin zu lassen - sind sehr ausgesprochen.

Erst nachdem sich eine gewisse Menge von Blut und Urin in der Blase angesammelt hat, die Blase nunmehr gewissermaßen entfaltet und die Rückwand dabei gedehnt wird, erst dann stellt sich die Bauchdeckenspannung als Zeichen der peritonealen Reizung ein. Sie verschwindet aber, sobald die Blase entleert zusammenfällt und so die Ursache der Reizung schwindet.

Allmählich kommt es zu einer mehr oder weniger ausgedehnten Urininfiltration in den submukösen Schichten der Blasenwand. Durch den Schleimhautriß und durch den Riß in der Muskelschicht sickert Urin durch die Muskelfasern hindurch bis in das subperitoneale Gewebe. Denn nur die intakte Blasenschleimhaut ist für Flüssigkeiten und Gifte undurchgängig. Die Blasenmuscularis und Subserosa sind nicht nur nicht undurchlässig, sondern gestatten auch eine gewisse Resorption. Sind inzwischen noch Bakterien in die Blase geschleppt - und dies geschieht, auch wenn man sich beim Katheterisieren der peinlichsten Asepsis befleißigt - so ist der Allgemeininfektion freie Bahn gegeben und es ist nicht wunderbar, daß man schon nach 36 Stunden die ersten Anzeigen der nahenden Peritonitis bemerkt. So sehen wir in obigem Falle, daß 48 Stunden nach dem Trauma eine exsudative Peritonitis besteht und daB die hintere Blasenwand in mehr als Talergröße nekrotisch, daß die Blasenserosa hier graugrünlich verfärbt und in leichte Falten gelegt ist. Bemerkenswert ist, daß der Riß nicht dem Centrum dieser nekrotisch-gangränösen Partie entspricht, daß er vielmehr im oberen medialen Quadranten liegt. Die Urin- 
infiltration und Nekrose hat sich nach unten und lateralwärts erstreckt.

Experimentell konnte v. St u be n ra u c $\mathrm{h}^{1}$ ) ganz ähnliche Erscheinungen erzeugen. Er sah, wie bei langsam steigendem Drucke in der Blase nach Verletzung der Schleimhaut Flüssigkeit zwischen die einzelnen Muskelbündel hindurchtritt und die Serosa abhebt.

Keine der geltenden Theorien über das Zustandekommen von Blasenrupturen gibt eine einwandfreie Erklärung für das Auftreten der subperitonealen Ruptur in obigem Falle. Es fehlt allerdings auch hier ein für das Zustandekommen der kompleten Blasenruptur wichtiger Faktor: die starke Füllung der Blase. Der Patient hatte nämlich eine halbe Stunde vor dem Trauma zuletzt Urin gelassen.

Nur der Umstand, daß der rechte Schambeinast zwei Sprunglinien aufwies und die Kleider des Patienten entsprechend dem rechten Beckenrand mit Straßenstaub bedeckt waren, lassen vermuten, in welcher Richtung die Gewalt gewirkt hat.

Nur ganz wenige Fälle von inkompleten Blasenrupturen sind beschrieben. Seld ow its $\mathrm{ch}^{2}$ ) berichtet über einen Fall:

Es handelte sich um eine Frau, die betrunken in Polizeigewahrsam kam und von da tags darauf in das Krankenhaus geschafft wurde. Das Gesicht war blaß und verfallen, Puls i20, Temperatur 38, Abdomen aufgetrieben, sehr schmerzhaft, gespannt. Dämpfung in beiden Lumbalgegenden. Mittelst Katheters werden I5o ccm Harn mit Blut entleert. In der Bauchhöhle fand sich blutig-eitriges Exsudat, besonders im Becken. Darmserosa injiziert. Am Blasenperitoneum alte Membranen. An der Blasenspitze eine talergroße, unregelmäßig konturierte Ruptur des Peritoneums. Die Muskelfasern sind stellenweise nur etwas eingerissen. Die Wunde geht nicht bis in das Blaseninnere. Tamponade der Blasengegend. Am 9. Tage geht der ganze Urin durch die Bauchwunde $a b$; diese ist septisch. Es zeigte sich bei der Sektion, daß auch die Blasenschleimhaut eingerissen war.

Es war also bei dem Trauma zu einem Riß der Blasen-Innenund Außenschicht gekommen, nur die Mittenschicht war unversehrt geblieben.

I) $\mathrm{Stubenra} \mathrm{u} \mathrm{ch,} \mathrm{Über} \mathrm{Festigkeıt} \mathrm{und} \mathrm{Elastizität} \mathrm{der} \mathrm{Harnblase.} \mathrm{Mit}$ Berücksichtigung der isol. traumat. Harnblasenzerreißung. Archiv f. klin. Chi rurgie, Bd. $5 \mathrm{I}$.

2) Seld owitsch, Archiv f. klin. Chirurgie 1904. 
Über einen zweiten Fall hat $\mathrm{K} u \mathrm{~h} \mathrm{n}^{1}$ ) berichtet.

Es handelt sich um einen alten Mann, der unter peritonitischen Erscheinungen in das Krankenhaus kam. Bei der Sektion zeigte sich am Blasenscheitel eine rundliche, mit zackigen Rändern begrenzte Offnung, welche Mucosa und Muscularis völlig durchsetzt und durch die man in einen dem Blasenscheitel links oben aufsitzenden, offenbar nur aus etwas verdicktem Peritoneum gebildeten halbkugeligen Sack gelangt, der mit Eiter gefiillt ist. Die äußere Peritonealfläche in dieser Gegend mit einer gelblich fibrinös-eitrigen Masse bedeckt, entspricht der Verklebungsstelle mit dem Dünndarm.

Die Ätiologie dieses Falles ist dunkel, es ist nicht ausgeschlossen, daß die Ruptur beim Katheterisieren gemacht wurde.

Die Prognose ist, wie diese beiden und der oben beschriebene dartun, recht schlecht. Im Handbuch der praktischen Chirurgie wird die Ansicht vertreten, daß die Prognose von der Beschaffenheit des Risses abhängt, und ob viel oder wenig Harn austritt. „,Ist der Riß nur klein, so sickert der Harn nur langsam heraus und hat Zeit, sich abzukapseln. Ist dagegen der Riß groß, so erfolgt die Urininfiltration in sehr ausgedehnter Weise, mit ihr zugleich eine Nekrose des lockeren Bindegewebes. Sie ist selbst imstande, das Peritoneum in Mitleidenschaft zu ziehen und eine diffuse Peritonitis hervorzurufen." Diese Ansicht erscheint mir zu optimistisch. Denn trotzdem in meinem Falle der Schleimhautriß nur I2 $\mathrm{mm}$ betrug, kam es leider zu keiner Abkapselung.

Glaubte man bisher bei inkompleten Blasenrupturen abwarten zu müssen und erst dann eingreifen zu dürfen, wenn bedrohlichere Erscheinungen auftreten, wenn die ersten Symptome der drohenden Peritonitis sich bemerkbar machen, so dürfte nach den obigen Ausführungen ein sofortiges operatives Eingreifen gerechtfertigt sein. Die Operation muBder Urininfiltration vorbeugen, und wenn sie schon besteht, sie zu beschränken suchen. Der Urininfiltration beugt man am zweckmäßigsten vor, wenn man so früh wie nur irgend möglich eingreift. Mein Chef, Herr Prof. Hermes, beabsichtigt in kommenden Fällen folgendermaßen vorzugehen: Sobald die Diagnose gesichert oder in hohem Grade wahrscheinlich ist, Freilegen der hinteren Blasenwand in steilster Beckenhochlagerung und Aufsuchen der lädierten resp.

I) K u h n, Fall von Harnblasenruptur. Münchn. med. Dissert. Igor. 
suffundierten Partie. Zeigt die hintere Blasenwand keine derartig veränderte Stelle, so ist die Blase an ihrer Vorderfläche zu eröffnen und ihre Innenfläche abzutasten und abzuleuchten. Sitzt der Schleimhaut-Muscularisriß so, daß man von der vorderen Incision bequem an ihn herankommt, so kann man ihn vom Blaseninneren aus zweischichtig nähen und die vordere Blasenwunde vollkommen schließen. Die Serosa über der Rißstelle wird durch mehrere Raffnähte eingestülpt. Die Peritonealwunde wird vollständig geschlossen, ebenso die Wunde an der Blasenvorderfläche. Nur in den prävesikalen Raum wird ein Gazestreifen gelegt. Ist es technisch nicht möglich, vom Blaseninneren aus die Rißstelle zu verschließen, so ist die inkomplete Ruptur in eine vollständige zu verwandeln, evt. etwas zu vergrößern und diese Wunde vom Bauch aus schichtweise zu verschließen. Auch hier wird die Serosa-Naht durch eine zweite Serosa-Raff-Naht versenkt und eingestülpt. In die letzte Serosa-Naht wird ein Zipfel des heruntergeholten Netzes eingeknüpft. Das Netz könnte auch, wie es L a nda u mit Recht für eitrige, resp. zweifelhafte gynäkologische Operationen empfiehlt, im kleinen Becken angeheftet werden. Es wird dadurch eine Barriere gegen eine Infektion der Darmserosa geschaffen und das kleine Becken von der übrigen Bauchhöhle abgeschlossen, also eine Extraperitonealisierung im weiteren Sinne. Die Bauchwunde könnte nun entweder vollkommen bis auf einen Streifen in dem Cavum Retzii geschlossen werden oder man könnte auch noch einen Tampon in den Douglas führen. Dieses Vorgehen gilt nur für die Fälle, wo keine oder nur eine minimale peritoneale Reizung besteht. - Weist die Serosa schon eine beginnende Gangrän auf, so muß man sich zur Excision der verfärbten Partie entschließen und durch schichtweise Naht den Defekt schließen. Die oben beschriebene Netzplastik dürfte zur Sicherung der Nahtlinie beitragen. Eine einfache Einstülpung und Übernähung der gangränösen Partie genügt obiger Voraussetzung nicht. Meistens wird man in diesem Stadium schon eine exsudative Peritonitis vorfinden. Hier wird der einzelne verschieden vorgehen, je nachdem er ein Anhänger des Spül- oder Trockenverfahrens ist.

Dieser Fall lehrt, daB die Diagnose inkomplete Blasenruptur in den ersten Stunden nach dem Unfall mit einer gewissen Sicher- 
heit zu stellen ist. Das klinische Bild der inkompleten Blasenruptur unterscheidet sich in den ersten Stunden nach dem Unfall durch das Fehlen der allgemeinen Bauchsymptome von dem der vollständigen Blasenruptur. Bei einem gewissen Füllungszustande der Blase tritt eine deutliche Bauchdeckenspannung auf, welche auf eine Reizung des Bauchfelles an der Blasenrißstelle hinweist, die aber nach Entleerung der Blase wieder verschwindet. In späteren Stunden (2. bis 3. Tag) verläuft die Krankheit unter dem Bilde der allgemeinen Bauchfellentzündung. Nur die Anamnese deutet den Ausgangspunkt der Bauchfellentzündung an. Möglichst frühzeitiger operativer Eingriff und Naht der Rißstelle beugen am sichersten der Urininfiltration der Blasenwand vor. 